\title{
REPRODUCIBILITY OF BRAIN-COGNITION RELATIONSHIPS USING DIFFERENT CORTICAL SURFACE-BASED ANALYSIS PROTOCOLS
}

\author{
${ }^{1}$ Kenia Martinez, ${ }^{2}$ Anand A. Joshi, ${ }^{3}$ Sarah K. Madsen, ${ }^{4}$ Shantanu Joshi, ${ }^{5}$ Sherif Karama, \\ ${ }^{1}$ Francisco J. Román, ${ }^{3}$ Julio Villalon-Reina, ${ }^{6}$ Miguel Burgaleta, ${ }^{3,4}$ Paul M. Thompson, ${ }^{1}$ Roberto Colom
} ${ }^{1}$ Facultad de Psicología, Universidad Autónoma de Madrid, Spain; ${ }^{2}$ Biomedical Imaging Group, University of Southern California, Los
Angeles, CA, USA; ${ }^{3}$ Imaging Genetics Center, Institute for Neuroimaging \& Informatics, USC Keck School of Medicine, Los Angeles,
CA $;{ }^{4}$ Department of Neurology, UCLA School of Medicine, Los Angeles, CA, USA; ${ }^{5}$ McGill University, Montreal, Quebec; ${ }^{6}$ Universitat
Pompeu Fabra, Barcelona, Spain

\begin{abstract}
Neuroimaging techniques are now widely used to understand relationships between brain features and cognitive performance. Nevertheless, studies do not always implicate the same anatomical neural networks in intellectual function. Here we used $\mathrm{T}_{1}$-weighted brain MRI scans obtained from a sample of 82 healthy young adults to study four potential sources of variability affecting the reproducibility of brain-cognition relationships: the neuroimaging protocol used, different measures of cortical gray matter, the nature of the cognitive measurement, and sample characteristics. We found that brain networks implicated in individual differences in cognition were not consistent when derived from different gray matter measures, or from different surface-based processing pipelines, even in equivalent samples of participants. Differences in the networks associated with cognition may reflect differences in the methods used to analyze them; in addition, different individuals may reach equivalent psychological goals through disparate brain networks.
\end{abstract}

Index Terms - Gray Matter Properties, Surface-Based Methods, Higher Order Cognition.

\section{INTRODUCTION}

The Parieto-Frontal Integration Theory (P-FIT) [1] is a framework for understanding the biological basis of human intelligence. Some researchers consider the findings to be unified, but others raise doubts about their consistency across studies. There are several potential explanations for the discrepancies. These include (1) the characteristics of the sample analyzed, (2) the methods for processing magnetic resonance images, (3) the brain features considered, and (4) the way in which intelligence is defined and measured. However, to the best of our knowledge, there is no research addressing the impact of these methodological variations in detail. Here we consider a comprehensive set of tests tapping key intelligence domains, namely fluid (Gf), crystallized-verbal $(G c)$ and spatial $(G v)$ intelligence.
Furthermore, we apply three surface-based protocols [CIVET, BrainSuite, and Cortical Pattern Matching (CPM)] for quantifying three gray matter properties: cortical thickness (CT), cortical surface area (CSA), and cortical gray matter volume $(\mathrm{CV})$. Afterwards, we analyzed the relationships between individual differences in psychological performance and the variability in the three morphological measures of the cortex. Finally, we systematically checked the reproducibility of findings across methods, gray matter tissue features, and samples.

Although this research is exploratory, general predictions could be made in advance based on previous findings and theory. First, the distribution and variability of each morphometric measure across the cortex, as well as more prominent brain correlates for each psychological factor, should be somewhat consistent across methods. Second, core regions underlying cognition, might be expected to emerge in matched samples. Also, each morphometric measure should show a similar pattern of distribution and variability across the cortex after accounting for the differences among surface-based protocols. Third, the variability in $\mathrm{CV}$ should be mainly driven by the variability in CSA, rather than in CT [2]. Finally, since psychometric estimates of cognitive performance at the level of intelligence first-order factors capture common variance shared by their respective tests, brain regions correlating with each latent construct will be mainly circumscribed to common brain regions that are involved in these tests.

\section{METHODS}

\subsection{Participants and Image Acquisition}

We studied 82 young adults ( 48 females, mean age $=19.9$ years, $\mathrm{SD}=1.5$ ). They were selected to exclude cases of medical or psychiatric illness. Informed consent was obtained, following the Helsinki guidelines.

MR images were obtained in a 3 T scanner (GE Healthcare) using a whole-body radiofrequency coil for signal excitation, and a quadrature 8-channel coil for reception. 3D $\mathrm{T}_{1}$ weighted scans were acquired in the sagittal plane with a SPGR sequence with the following parameters: TR $=6.8$ $\mathrm{ms}, \mathrm{TE}=3.1 \mathrm{~ms}$, Preparation Time $=750 \mathrm{~ms}$; flip angle $=$ 
$12^{\circ} ; 1 \mathrm{~mm}$ slice thickness, $288 \times 288$ acquisition matrix $(0.8 \times 0.8 \times 1 \mathrm{~mm}$ voxel size $), 512 \times 512$ reconstructed matrix (0.47x0.47x1 mm voxel size), $240 \mathrm{~mm}$ FOV and 196 images (slices) per acquisition.

\subsection{Psychological measures}

Nine cognitive tests were administered to measure the psychological constructs of interest. Importantly, each cognitive domain was evaluated using three different measures to obtain theoretically representative scores.

Gf was measured using the Advanced Progressive Matrices Test [3], the abstract reasoning subtests from the Differential Aptitude Test (DAT-AR) battery [4], and the inductive reasoning subtests from the Primary Mental Abilities (PMA-R) battery [5]. Gc was measured by DATVR (verbal reasoning), DAT-NR (numerical reasoning), and PMA-V (vocabulary). $G v$ was measured by DAT-SR (spatial relations), PMA-S (mental rotation), and the rotation of solid figures test [6].

\subsection{MRI data processing: Surface-Based Processing Protocols}

Three different processing protocols were applied to obtain three different gray matter indices. Table 1 gives a comparative overview of procedures and tools used for each protocol. This table reveals commonalities among methods and the algorithms involved at each processing step. The pipelines only agree in the size of the smoothing kernel. Although prior research suggests that the optimal size of smoothing filters is not the same for different morphometric measures (as it depends on the scale of the effect of interest), there is not a consensus regarding values that would be most appropriate for a given application [7]. Therefore, the same kernel size $(10 \mathrm{~mm})$ was used for filtering the gray matter properties considered, to make them more comparable.

Gray matter measures were computed using the subjects' volumetric images without rescaling. The tools and procedures employed in the remaining steps are sometimes shared by two pipelines, such as MINC tools for volumetric spatial normalization and intensity normalization; algorithms included in BrainSuite software [http://neuroimage.usc.edu/neuro/BrainSuite] to perform brain extraction and tissue classification; and the $t_{\text {link }}$ metric to compute CT. However, pipelines differ completely in the algorithms for reconstructing and align the cortical surfaces, and in the methods used for CSA and CV measurement. Finally, note that CSA and CV were obtained from CIVET and BrainSuite pipelines only, since the CPM protocol [8] does not directly measure these two indices.

\begin{tabular}{|c|c|c|c|}
\cline { 2 - 4 } \multicolumn{1}{c|}{} & CIVET & BrainSuite & CPM \\
\hline Steps & $\begin{array}{c}\text { Procedure/ } \\
\text { Software/Tool }\end{array}$ & $\begin{array}{c}\text { Procedure/ } \\
\text { Software/Tool }\end{array}$ & $\begin{array}{c}\text { Procedure/ } \\
\text { Software/Tool }\end{array}$ \\
\hline
\end{tabular}

\begin{tabular}{|c|c|c|c|}
\hline 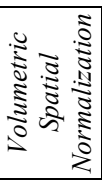 & $\begin{array}{l}\text { Linear registration } \\
\text { to ICBM } 152 \text { using } \\
\text { with } 9 \text { parameters } \\
\text { using MINC tools }\end{array}$ & $\begin{array}{l}\text { Linear registration to } \\
\text { Colin27 with } 6 \\
\text { parameters using FSL } \\
\text { tools }\end{array}$ & $\begin{array}{l}\text { Linear registration to } \\
\text { ICBM } 53 \text { with } 9 \\
\text { parameters using } \\
\text { MINC tools }\end{array}$ \\
\hline 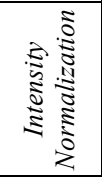 & $\begin{array}{l}\text { N3 (from MINC } \\
\text { toolbox }\end{array}$ & $\begin{array}{c}\text { BFC } \\
\text { (BrainSuite software) }\end{array}$ & $\begin{array}{l}\text { N3 from MINC } \\
\text { toolbox }\end{array}$ \\
\hline 语 & $\begin{array}{l}\text { BET using FSL } \\
\text { tools }\end{array}$ & $\begin{array}{l}\text { BSE (BrainSuite } \\
\text { software). Manual } \\
\text { editing of the brain } \\
\text { mask to improve the } \\
\text { brain extraction }\end{array}$ & $\begin{array}{l}\text { BSE (BrainSuite } \\
\text { software). Manual } \\
\text { editing of the brain } \\
\text { mask to improve the } \\
\text { brain extraction }\end{array}$ \\
\hline 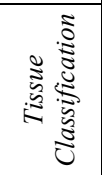 & $\begin{array}{c}\text { INSECT } \\
\text { (CIVET pipeline, } \\
\text { version 1.1.9) }\end{array}$ & $\begin{array}{c}\text { PVC } \\
\text { (BrainSuite software) }\end{array}$ & $\begin{array}{c}\text { PVC } \\
\text { (BrainSuite } \\
\text { software) }\end{array}$ \\
\hline 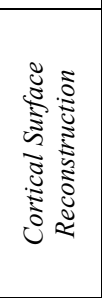 & $\begin{array}{c}\text { CLASP } \\
\text { implemented in } \\
\text { CIVET pipeline } \\
\text { (version 1.1.9). The } \\
\text { resulting } \\
\text { hemispheric mesh } \\
\text { has } 81924 \text { triangles } \\
\text { and } 40962 \text { vertices } \\
\text { for all subjects }\end{array}$ & $\begin{array}{l}\text { Cortical Surface } \\
\text { Extraction algorithm } \\
\text { implemented in } \\
\text { BrainSuite software. } \\
\text { The resulting whole } \\
\text { brain mesh has } \\
\text { approximately } 500000 \\
\text { vertices depending on } \\
\text { the subject's brain }\end{array}$ & $\begin{array}{l}\text { MSD. The resulting } \\
\text { hemispheric mesh } \\
\text { has } 131072 \text { triangles } \\
\text { and } 65536 \text { vertices } \\
\text { for all subjects }\end{array}$ \\
\hline 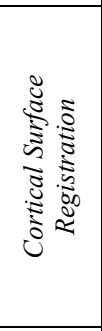 & $\begin{array}{c}\text { Non-linear } \\
\text { hierarchical } \\
\text { deformable } \\
\text { registration to a } \\
\text { high-resolution } \\
\text { average template } \\
\text { based on depth } \\
\text { potential function } \\
\text { (CIVET pipeline } \\
\text { version 1.1.9). } \\
\end{array}$ & $\begin{array}{l}\text { Non-linear registration } \\
\text { to a high-resolution } \\
\text { template based on } \\
\text { curvature implemented } \\
\text { as SVREG toolbox [9] } \\
\text { in BrainSuite software. }\end{array}$ & $\begin{array}{l}\text { Diffeomorphic sulcal } \\
\text { shape matching } \\
\text { across subjects [10] }\end{array}$ \\
\hline 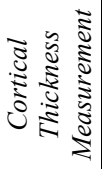 & $t_{\text {link }}$ metric & $t_{\text {link }}$ metric & 3D Eikonal equation \\
\hline 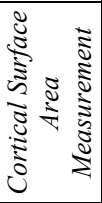 & $\begin{array}{l}\text { Sum of the area of } \\
\text { the } 6 \text { triangles } \\
\text { linked to each } \\
\text { vertex divided by } 6 \\
\text { (computed on the } \\
\text { medial surface) }\end{array}$ & $\begin{array}{l}\text { Assigning a portion of } \\
\text { each triangular area to } \\
\text { the vertex with the } \\
\text { Voronoi's method } \\
\text { (computed on the } \\
\text { medial surface) }\end{array}$ & \\
\hline 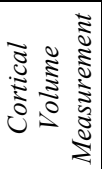 & $\begin{array}{l}\text { Volume integral of } \\
\text { the prism }\end{array}$ & $\begin{array}{l}\text { Multiplying the surface } \\
\text { area at each vertex by } \\
\text { its thickness }\end{array}$ & \\
\hline
\end{tabular}

Table 1. Key surface-based processing steps and tools used in each protocol considered. N3=Nonparametric Non-uniform Intensity Normalization [11]. BFC $=$ Bias Field Corrector [12]. BET $=$ Brain Extraction Tool [13]. BSE=Brain Surface Extractor [12]. INSECT=Intensity Normalized Stereotaxic Environment for Classification of Tissues [14]. $\mathrm{PVC}=$ Partial Volume Classifier [12]. CLASP $=$ Constrained Laplacian-based ASP [15]. MSD=Multiple Surface Deformation [16].

\subsection{Statistical Analysis}

Statistical analyses were implemented using SurfStat [http://www.math.mcgill.ca/keith/surfstat/]. Firstly, the 
distribution and variability of each morphometric measure was analyzed by computing the mean and standard deviation at each cortical point. Second, to study relationships between psychological factors and gray matter properties, each morphometric measure was set as the dependent variable in a linear multiple regression model with four predictors: sex, age, handedness, and the corresponding cognitive factor/test. These analyses were computed at vertex level for the whole sample $(n=82)$ and for two equivalent subsamples $(\mathrm{n}=41)$. Lastly, Student's $t$, Pearson's $r$ and $p$-values maps for the main effect of each cognitive regressor $\left(b_{l}\right)$ on each morphological index were obtained. These uncorrected statistical maps were used to analyze the spatial patterns of brain-cognition relationships. Also, tvalue threshold of statistical significance was established, taking into account multiple comparisons $(\alpha=0.05)$ via the False Discovery Rate (FDR) method [17]. The statistical power associated to different effects and sample sizes was estimated using $G^{*}$ Power software.

\section{RESULTS AND DISCUSSION}

\subsection{How Do Different Surface-Based Protocols Affect Agreement Across Studies?}

We did not find strong convergence among results derived from the three difference surface-based analysis protocols.

First, and especially important, the general pattern of highest and lowest values computed for CT, CSA, and CV across the cortex, as well as the variability maps across subjects, did not converge across protocol, regardless of the gray matter measure considered. For CT, the average and distribution of CT derived from the CPM approach agree with postmortem data [18] in more extent than those derived from BrainSuite and CIVET pipelines [Figure 1]. Further, consistent with histological studies, CT obtained using the CPM protocol showed more variance across individual brains in heterotypical than in homotypical cortical regions. Second, the patterns of $\mathrm{CV}$ and their relationship to measures of cognition were mainly driven by the patterns in CSA, rather than in CT [Figure 2]. Further, CSA and CT poorly correlated, regardless of the applied surface-based protocol (mean correlation values using CIVET $=0.004$ and BrainSuite $=0.11)$. Third, there was no strong agreement among methods in the pattern of associations observed between morphological measures across the cortex and cognitive factors: peak values do not overlap for the different morphological measures considered. [e.g.Figure3].

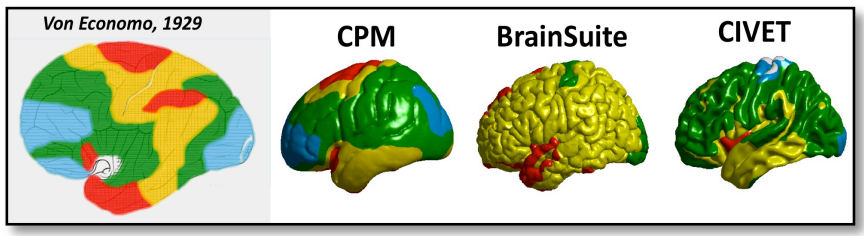

Figure 1. Comparison of CT distribution maps derived from postmortem and neuroimaging data (left lateral view; $\mathrm{n}=82$ ).
Perhaps surprisingly, equivalent brain regions exhibit opposite directions in their relationship to cognitive factors depending on the surface-based protocol. Statistical maps did not survive correction for multiple comparisons (FDR; $\alpha=0.05$ ), so reported results derived from each of the considered protocols were uncorrected (more lenient threshold $=p<0.005$ ). This could explain the lack of convergence, as these results were not robust enough to be statistically significant after FDR correction.

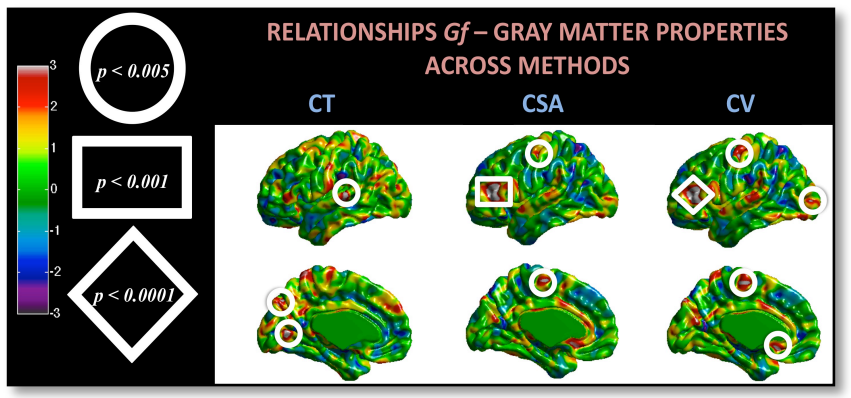

Figure 2. $t$-maps for the effect of $G f$ on CT, CSA and CV obtained by CIVET pipeline (left hemisphere; $n=82$ ). Circles, squares and rhombus are highlighting significant brain correlates at three commonly used uncorrected statistical thresholds: $0.005,0.001$ and 0.0001 respectively.

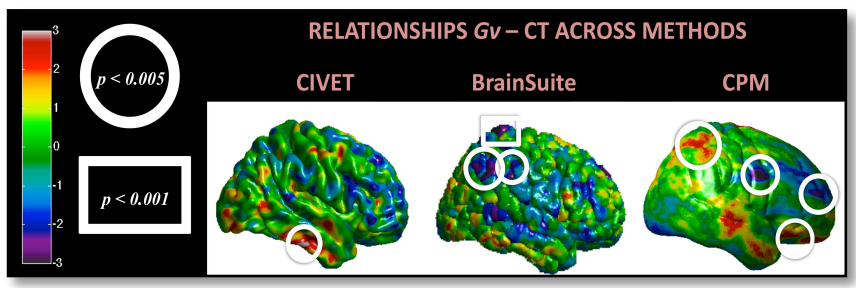

Figure 3. $t$-maps for the effect of $G v$ on CT obtained by different surfacebased protocols. Circles and squares are highlighting significant brain correlates at two commonly used uncorrected statistical thresholds: 0.005 and 0.001 respectively. Only left lateral view is displayed.

\subsection{Are Psychologically Matched Samples show similar Patterns of Anatomical Correlates of Intellectual Function?}

The straight answer is 'no', they are not. Even when the methods were consistent regarding their potential bias when estimating each gray matter index (patterns of distribution and variability are the same for the considered subsamples within a given protocol; e.g. Figure 4-A), the brain correlates for psychologically equivalent samples failed to converge, suggesting a broad inter-subject variability in the brain networks supporting intelligence.

We applied an analytical approach to understand inconsistencies in the literature related to the variability of different samples of subjects. The overall cohort was divided into two independent subsamples matched with respect to all cognitive domains, sex, age, and handedness. This was intended to maximize the likelihood of getting replication, but even here, convergence across methods was not achieved.

Uncorrected significant results $(p<0.005)$ for the smaller subsamples showed higher effect sizes ( $r$-values) than those 
found for the complete group, but any statistical map survived FDR correction.

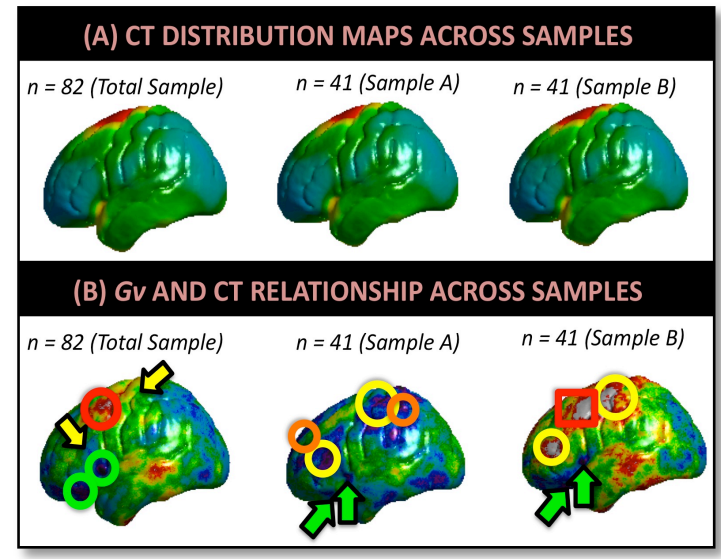

Figure 4. (A) Comparison of CT distribution derived from LONI pipeline across samples. (B) $t$-maps for the effect of $G v$ on CT across samples. Circles and squares are signaling results significant at $\mathrm{p}<0.005$ and $\mathrm{p}<0.001$ respectively. Only left lateral view is displayed.

Statistical power depends on sample size and the expected effect sizes in the populations. Keeping the threshold for significance constant, if the effect size is strong enough, a small sample size can offer sufficient statistical power. In fact, most of the prominent findings for the complete group were a composite of those obtained in both subsamples, irrespective of the imaging method or the gray matter property analyzed [e.g. Figure 4-B]. Thus, some effects became significant by increasing sample size (green circles and arrows, Figure 4), but others became non-significant or lower. This last phenomenon occurred if the discovered effect in the complete group was only supported by one of the subsamples (red circle and square, Figure 4), or when the relationship between the psychological factor and a given morphometric measure was detected for both subsamples, but in opposite directions (yellow circles and arrows, Figure 4).

\subsection{How do the chosen cognitive tests influence the results?}

As predicted, anatomical correlates of each latent cognitive construct summarize the overlap among their respective measures [Figure 5]. Also, results for specific intelligence tests are widespread across the cortex and they show only minor overlaps with their respective factors. So, finegrained psychometric approaches for defining the constructs of interest are recommended, since single cognitive measures provide different results at biological level.

\section{CONCLUSION}

In closing, the present study reveals a substantial vulnerability in neuroimaging studies likely attributable to methodological sources of instability. This is consistent with the published evidence that do not always implicate the

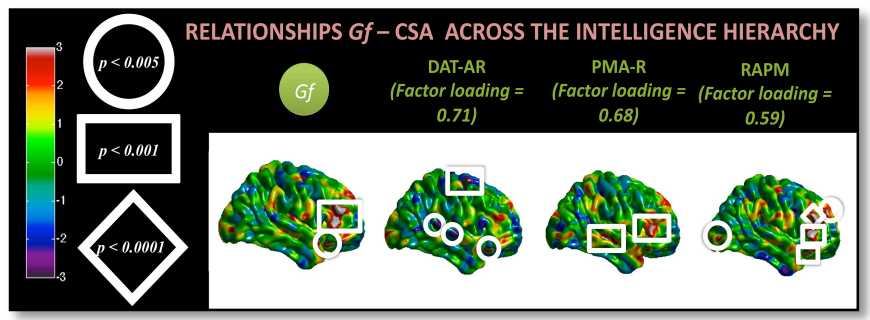

Figure 5. $t$-maps for the effect of $G f$ and its corresponding tests on CSA obtained by CIVET pipeline (right hemisphere lateral view; $n=82$ ). Circles, squares and rhombus are highlighting significant brain correlates at three commonly used uncorrected statistical thresholds: $0.005,0.001$ and 0.0001 respectively.

same regions when different imaging protocols, samples, or cognitive measures are used. Nevertheless, we strongly think there are reasons to be optimistic. Acknowledging these challenges is the first step towards addressing the problematic aspects of current scientific procedures and identifying efficient solutions. Fixing the observed problems not only involves improvements in the software for processing neuroimaging data. Maybe our main challenge is to rethink the way in which we are studying complex psychological factors at the biological level. It has been suggested [19] that "not all brains work in the same way" and this might imply that different individuals reach equivalent complex psychological goals by different brain networks. Averaging anatomical data may help to overcome the revealed inconsistencies, but this latter strategy might mask relevant information for a proper understanding. Actually, the positive discoveries presented here did not survive to correction by multiple comparisons, so maybe all what we found is spurious in terms of brain properties underlying cognition. If this is indeed the case, how to expect replication across neuroimaging methods or samples?

\section{REFERENCES}

[1]Jung RE. \& Haier RJ. The Parieto-Frontal Integration Theory (PFIT) of intelligence: converging neuroimaging evidence. Behavioral and Brain Sciences, 30(02), 135-154, 2007.

[2]Winkler AM, Kochunov P, Blangero J, Almasy L, Zilles K, Fox PT., ... \& Glahn, DC. Cortical thickness or grey matter volume? The importance of selecting the phenotype for imaging genetics studies. Neuroimage, 53(3), 1135-1146, 2010.

[3]Raven J, Raven JC, \& Court JH. Manual for Raven's Progressive Matrices and Vocabulary Scales. San Antonio, TX, Pearson Assessment, 2004.

[4]Bennett GK, Seashore HG, \& Wesman AG. Differential Aptitude Test (DAT-5). Madrid, TEA, S.A, 2005.

[5]Thurstone L. Primary mental abilities. Psychometric Monographs, $1,1938$.

[6]Yela, M. Rotación de Figuras Macizas. TEA, Madrid, 1969

[7]Zhao L, Boucher M, Rosa-Neto P, \& Evans AC. Impact of scale space search on age-and gender-related changes in MRI-based cortical morphometry. Human Brain Mapping, 2012

[8]Thompson PM, Hayashi KM, Sowell ER, Gogtay N, Giedd JN, Rapoport JL, de Zubicaray GI, Janke AL, Rose SE, Semple J, Doddrell DM, Wang YL, van Erp TGM, Cannon TD, Toga AW (2004). Mapping Cortical Change in Alzheimer's Disease, Brain Development, and Schizophrenia, Special Issue on Mathematics in Brain Imaging 
(Thompson PM, Miller MI, Ratnanather JT, Poldrack R, Nichols TE, eds.), NeuroImage, 23 Suppl 1:S2-18, September 2004.

[9]Joshi AA., Shattuck, DW, \& Leahy RM. A method for automated cortical surface registration and labeling. In Biomedical Image Registration (pp. 180-189). Springer Berlin Heidelberg, 2012.

[10]Joshi, SH., Cabeen RP., Joshi AA., Sun B, Dinov I, Narr KL, ... \& Woods RP. Diffeomorphic sulcal shape analysis on the cortex. Medical Imaging. IEEE Transactions on, 31(6), 1195-1212, 2012.

[11]Sled JG, Zijdenbos AP, \& Evans, AC. A nonparametric method for automatic correction of intensity nonuniformity in MRI data. Medical Imaging, IEEE Transactions on, 17(1), 87-97, 1998.

[12]Shattuck D, Sandor-Leahy S, Schaper K, Rottenberg DA, Leahy RM. Magnetic resonance image tissue classification using a partial volume model. Neuroimage, 13 (5), 856-876, 2001.

[13]Smith SM. Fast robust automated brain extraction. Human Brain Mapping, 17(3), 143-155, 2002.

[14]Zijdenbos A, Forghani R, \& Evans A. Automatic quantification of MS lesions in 3D MRI brain data sets: validation of INSECT. In Medical Image Computing and Computer-Assisted InterventationMICCAI'98 (pp. 439-448). Springer Berlin Heidelberg, 1998.

[15] Kim, JS, Singh, V, Lee JK, Lerch J, Ad-Dab'bagh Y, MacDonald $\mathrm{D}, \ldots$ \& Evans AC. Automated 3-D extraction and evaluation of the inner and outer cortical surfaces using a Laplacian map and partial volume effect classification. Neuroimage. 27(1), 210-221, 2005.

[16]MacDonald D, Avis D, \& Evans AC. Multiple surface identification and matching in magnetic resonance images. In Visualization in Biomedical Computing 1994 (pp. 160-169).

[17]Genovese CR, Lazar N, Nichols TE. Thresholding of Statistical Maps in Functional Neuroimaging Using the False Discovery Rate. NeuroImage, 15, 870-878, 2002.

[18] von Economo, C. F. (1929). The cytoarchitectonics of the human cerebral cortex. Humphrey Milford University Press.

[19] Haier RJ, Jung RE, Yeo RA, Head K, \& Alkire MT. Structural brain variation and general intelligence. NeuroImage, 23(1), 425-433, 2004. 